\title{
Conformité des bilans comparatifs des médicaments à l'admission transmis au département de pharmacie par télécopieur
}

\author{
Sophie Penfornis, Pascal Bédard, Benoit Bailey et Jean-François Bussières
}

\section{INTRODUCTION}

$\mathrm{L}$ e bilan comparatif des médicaments (BCM) est un processus

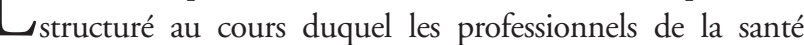
travaillent en partenariat avec les patients, les familles et les soignants pour assurer la transmission d'une information exacte et complète sur les médicaments aux points de transition des soins du patient (c.-̀̀-d. admission, transfert et congé). Le processus englobe la production d'une liste complète de tous les médicaments que le patient prenait avant l'admission, c'est-àdire le meilleur schéma thérapeutique possible. Cette liste est établie grâce à diverses sources et comprend des renseignements sur les médicaments d'ordonnance, les médicaments en vente libre, les vitamines et suppléments, ainsi que le nom du médicament, la dose, la fréquence et la voie d'administration. Toute anomalie relevée entre ce qui est prescrit au patient et ce qu'il prend en réalité sera signalée au fournisseur de soins pour être corrigée.

Létablissement d'un plan écrit pour instaurer le BCM partout dans l'organisme de soins est un test de conformité établi dans l'intitulé de la Pratique organisationnelle requise par Agrément Canada ${ }^{1}$. Ce plan doit comprendre les endroits et les échéances prévus pour l'établissement du BCM ${ }^{1}$. L'Organisation mondiale de la santé a également élaboré un protocole opératoire normalisé pour l'établissement de cette démarche ${ }^{2,3}$, tout comme la Haute Autorité de Santé en Francé.

Jusqu'à $60 \%$ de divergences ont été recensées lors de la démarche du BCM à l'admission, au transfert ou au congé d'un patient hospitalisé et jusqu’à $6 \%$ des patients présentant une divergence ont subi une conséquence néfaste suite à la cessation par inadvertance de la prescription d'un médicament ${ }^{6}$. Environ $50 \%$ des erreurs médicamenteuses observées à l'hôpital auraient pu être prévenues ${ }^{7,8}$ et plusieurs études ont démontré l'efficacité de la démarche du BCM pour prévenir et réduire les erreurs médicamenteuses dans le cadre d'un séjour à l'hôpital ${ }^{9-11}$. La priorité de la campagne "Soins de santé plus sécuritaires maintenant! » de l'Association canadienne des centres de santé pédiatriques en 2005 visait justement l'établissement de BCM dans la population pédiatrique ${ }^{12}$.

Le pharmacien est un acteur de choix pour recueillir la liste des médicaments pris par le patient et déceler les divergences, mais compte tenu des ressources disponibles, ce scénario est souvent inapplicable à tous les patients vus ou admis dans un hôpital ${ }^{13-15}$. Au besoin, les parents, assistants techniques en pharmacie, infirmières et médecins peuvent participer à la rédaction d'un $\mathrm{BCM}^{16-19}$. Les difficultés relatives à l'implantation d'une démarche pour la mise en place d'un BCM comportent notamment la pénurie de ressources humaines, la charge de travail et les confusions de rôle ${ }^{20}$.

Dans l'optique d'améliorer l'utilisation et la conformité du BCM à l'admission et de se conformer aux Pratiques organisationnelles requises, nous avons introduit l'obligation de transmettre le BCM à l'admission par télécopieur au département de pharmacie. L'envoi des BCM à la pharmacie permettait de quantifier le nombre de BCM remplis et reçus et $\mathrm{y}$ donnait accès en temps réel à tout le personnel du département de pharmacie. Ainsi, le système mis en place permet d'accéder à l'information non seulement lorsqu'il y a présence de pharmaciens décentralisés aux étages mais en tout temps. Lobjectif principal consistait à évaluer la proportion d'admissions de patients aux unités de soins, comportant un BCM conforme. L'objectif secondaire visait à mesurer les retombées de la révision du format du BCM à l'admission sur sa conformité.

\section{DESCRIPTION DE LA PRATIQUE}

Il s'agit d'une étude pilote descriptive. À partir du 6 décembre 2010, tous les BCM à l'admission des patients devaient être télécopiés à la pharmacie. Le BCM à l'admission pouvait être rempli par le parent, l'infirmière, le pharmacien (ou le résident ou l'étudiant en pharmacie) ou le médecin (ou 
le résident ou l'étudiant en médecine). Les BCM à l'admission étaient télécopiés à un numéro distinct de celui utilisé pour l'envoi des ordonnances afin d'évaluer la charge de travail et la faisabilité de l'intégration de cette tâche aux activités courantes du département de pharmacie. Le personnel en a été avisé officiellement par un envoi électronique aux cadres et verbalement lors des tournées quotidiennes de notre équipe d'implantation.

Le pharmacien recevant la télécopie du BCM à l'admission devait confirmer l'association du document numérique au bon patient, réalisée par le personnel technique, et utiliser, dans la mesure du possible, l'information contenue dans le BCM à l'admission en présence de divergences par rapport aux ordonnances de la première journée de séjour hospitalier.

Le formulaire du BCM utilisé était en vigueur depuis 2008 (Annexe 1, publié au www.cjhp-online.ca/index.php/cjhp/ issue/view/87/showToc). Une nouvelle version du BCM, disponible à partir du 11 janvier 2011, prenait en compte la mise en place de l'envoi électronique et des commentaires du personnel soignant (Annexe 2, publié au www.cjhponline.ca/index.php/cjhp/issue/view/87/showToc). Cette version révisée comportait notamment des cases à cocher pour les sources de l'histoire médicamenteuse, ainsi que des espaces désignés pour inscrire le nom du médicament, la dose, la voie et l'horaire d'administration ainsi que le moment de la dernière prise.

Les admissions des mères et des enfants ayant séjourné dans notre institution du 6 décembre 2010 au 6 février 2011 ont été incluses dans l'étude. Les admissions en oncologie ont été exclues, puisque la plupart des médicaments utilisés pour les patients ambulatoires étaient dispensés par la pharmacie satellite externe à notre établissement plutôt que par la pharmacie communautaire et que l'équipe de pharmaciens en oncologie élaborait le BCM à l'admission à partir du dossier informatisé complet.

La conformité des BCM à l'admission dûment remplis a été évaluée de façon globale (c.-à-d. toutes les sections du formulaire) et spécifique (c.-à-d. critères de la section médicament). Une variable était considérée conforme si l'information requise était colligée dans la section appropriée ou si une mention équivalente à "aucune donnée disponible» était inscrite. Notons toutefois que, dans le cadre de cette étude pilote, la qualité de l'information n'a pas été évaluée par rapport au dossier pharmacologique en vigueur auprès des pharmaciens communautaires.

La proportion des admissions comportant un BCM a été calculée globalement ainsi que par unité de soins. Les deux versions du BCM à l'admission ont également été comparées afin de mesurer les retombées de la révision du format du BCM sur sa conformité. Des statistiques descriptives sont rapportées.

\section{RÉSULTATS}

Durant la période de deux mois qu'a duré l'étude, il y a eu un total de 2456 admissions aux unités de soins concernées par le BCM. En moyenne, 28 \% des admissions comportaient un BCM à l'admission ayant été transmis, des résultats qui

\section{Tableau 1. Proportion de BCM reçus par admission et par unité de soins}

\begin{tabular}{|c|c|c|c|c|c|c|}
\hline \multirow[b]{2}{*}{ Unités de soins* } & \multicolumn{2}{|c|}{$\begin{array}{c}\text { De décembre } 2010 \text { à } \\
\text { février } 2011\end{array}$} & \multicolumn{2}{|c|}{ Novembre $2008 \dagger$} & \multicolumn{2}{|c|}{ Avril 2008t } \\
\hline & $\begin{array}{l}\text { Admissions } \\
\text { (n) }\end{array}$ & $\begin{array}{c}\text { Admissions } \\
\text { avec BCM } \\
(\%)\end{array}$ & $\begin{array}{l}\text { Admissions } \\
\text { (n) }\end{array}$ & $\begin{array}{c}\text { Admissions } \\
\text { avec BCM } \\
(\%)\end{array}$ & $\begin{array}{l}\text { Admissions } \\
\text { (n) }\end{array}$ & $\begin{array}{c}\text { Admissions } \\
\text { avec BCM } \\
(\%)\end{array}$ \\
\hline Psychiatrie & 20 & 55 & $\mathrm{NE}$ & $\mathrm{NE}$ & 15 & 0 \\
\hline Pédiatrie-A & 180 & 50 & 11 & 46 & 12 & 50 \\
\hline Pédiatrie-B & 159 & 41 & 21 & 24 & 24 & 63 \\
\hline Multispécialités-A & 124 & 37 & 2 & 100 & 19 & 0 \\
\hline Pédiatrie-C & 194 & 35 & 13 & 54 & 13 & 85 \\
\hline Multispécialités-B & 72 & 22 & NE & NE & NE & NE \\
\hline Obstétrique & 737 & 18 & 44 & 64 & 89 & 69 \\
\hline Pédiatrie-D & 43 & 14 & 13 & 15 & 8 & 0 \\
\hline Gynécologie & 209 & 13 & 17 & 41 & 20 & 60 \\
\hline Chirurgie-A & 305 & 5 & 48 & 70 & 40 & 17 \\
\hline Chirurgie-B & 201 & 4 & & & & \\
\hline Infectiologie & 137 & 1 & 10 & 90 & 11 & 82 \\
\hline Soins intensifs & 75 & 0 & 13 & 23 & 15 & 7 \\
\hline Globalement & 2456 & 28 & 192 & 53 & 266 & 46 \\
\hline $\begin{array}{l}\text { BCM = bilan comp } \\
\text { *Certaines unités d } \\
\text { (p.ex. Pédiatrie-A). } \\
\text { †Les évaluations d'a } \\
\text { les unités de psychi } \\
\text { l'étude. }\end{array}$ & $\begin{array}{l}\text { nts, } \mathrm{NE}=\mathrm{no} \\
\text { uées en sous } \\
2008 \text { ont é }\end{array}$ & $\begin{array}{l}\text { évalué. } \\
\text { ctions selon I } \\
\text { réalisées en p } \\
\text { ient pas la pl }\end{array}$ & spécialité. C€ & us-sections & t représenté & $\begin{array}{l}\text { ar des lettres } \\
\text { s. Seules } \\
\text { sation de }\end{array}$ \\
\hline
\end{tabular}


different selon les unités de soins, soit de $0 \%$ aux soins intensifs à $55 \%$ en psychiatrie (tableau 1). Ces BCM à l'admission comportaient la mention de 1057 médicaments.

Les améliorations apportées à la version révisée du formulaire de $\mathrm{BCM}$ ont contribué à une augmentation de la conformité des éléments suivants : statut allergique ( $84 \%$, version originale c. $92 \%$, version révisée), personne ressource consultée (19\% c. $52 \%)$, mention de produits de santé naturels (31\% c. $46 \%$ ), dose de médicament ( $42 \%$ c. $59 \%)$, voie (15\% c. $62 \%)$, horaire d'administration (53\% c. $66 \%$ ), date/heure de la dernière prise (2\% c. $39 \%$ ) (tableau 2 ).

\section{Tableau 2. Conformité des variables des BCM}

\begin{tabular}{|c|c|c|c|c|}
\hline \multirow[b]{2}{*}{ Variable } & \multicolumn{4}{|c|}{ Conformité, $n(\%)$} \\
\hline & \multirow{2}{*}{\multicolumn{2}{|c|}{$\begin{array}{l}\text { Version originale } \\
n=565 \text { patients }\end{array}$}} & \multirow{2}{*}{\multicolumn{2}{|c|}{$\begin{array}{c}\text { Version révisée } \\
n=130 \text { patients }\end{array}$}} \\
\hline & & & & \\
\hline Statut allergique & 476 & $(84)$ & 120 & (92) \\
\hline Statut intolérance & 192 & (34) & 46 & (35) \\
\hline Poids & 199 & (35) & NA & \\
\hline Taille & 99 & (18) & NA & \\
\hline Surface corporelle & 1 & $(<1)$ & NA & \\
\hline Personne ressource consultée & 107 & (19) & 67 & (52) \\
\hline Diagnostic(s) & 180 & (32) & NA & \\
\hline Médicament(s) & 549 & (97) & 120 & (92) \\
\hline Produit(s) de santé naturel(s) & 175 & (31) & 60 & (46) \\
\hline Nom de la pharmacie communautaire & 1 & $(<1)$ & 2 & $(2)$ \\
\hline Numéro de téléphone de la pharmacie communautaire & 1 & $(<1)$ & 2 & (2) \\
\hline Pharmacien communautaire contacté & 0 & $(0)$ & 0 & (0) \\
\hline Autres informations pertinentes & 62 & (11) & NA & \\
\hline Signature du patient/parent/tuteur & 187 & (33) & 26 & (20) \\
\hline Signature du professionnel désigné & 351 & (62) & NA & \\
\hline Signature d'un $2^{e}$ professionnel & 55 & (10) & 106 & (82) \\
\hline signature d'un $3^{e}$ professionnel & 7 & $(1)$ & 4 & (3) \\
\hline Date/heure de la signature du patient/parent/tuteur & 164 & (29) & 22 & (17) \\
\hline Date/heure de la signature du professionnel désigné & 332 & (59) & NA & \\
\hline Date/heure de la $2^{\mathrm{e}}$ signature & 51 & (9) & 102 & (78) \\
\hline Date/heure de la $3^{\text {e }}$ signature & 3 & (1) & 3 & (2) \\
\hline Paraphe patient/parent/tuteur & 95 & (17) & 19 & (15) \\
\hline Paraphe du professionnel désigné & 303 & (54) & NA & \\
\hline Paraphe du $2^{\mathrm{e}}$ professionnel & 45 & (8) & 97 & (75) \\
\hline Paraphe du $3^{e}$ professionnel & 5 & (1) & 3 & (2) \\
\hline Intention médicale confirmée & 13 & (2) & NA & \\
\hline Section médicaments & $n=853 \mathrm{me}$ & licaments & $n=204 \mathrm{~m}$ & licaments \\
\hline Nom & 703 & $(82)$ & 160 & (78) \\
\hline Dose & 360 & $(42)$ & 121 & (59) \\
\hline Voie & 129 & (15) & 127 & (62) \\
\hline Horaire d'administration & 453 & (53) & 134 & (66) \\
\hline Date/heure de dernière prise & 15 & (2) & 80 & (39) \\
\hline $\begin{array}{l}\text { Médicaments dont le nom est non conforme } \\
\text { (p. ex. orthographe) mais jugé compréhensible }\end{array}$ & 41 & (5) & 15 & (7) \\
\hline
\end{tabular}

La figure 1 illustre la proportion du nombre de médicaments par BCM à l'admission. La majorité des patients n'avaient à leur BCM aucun médicament (40\%) ou avaient un médicament (24\%) par BCM.

\section{DISCUSSION}

Malgré la présence répandue des pharmaciens canadiens soient présents de façon répandue dans les programmes de soins, c'est-à-dire au moins un pharmacien dans un secteur d'hospitalisation dans $89 \%$ des $\operatorname{cas}^{21}$, les pharmaciens assignés à la validation centralisée des ordonnances n'ont généralement 


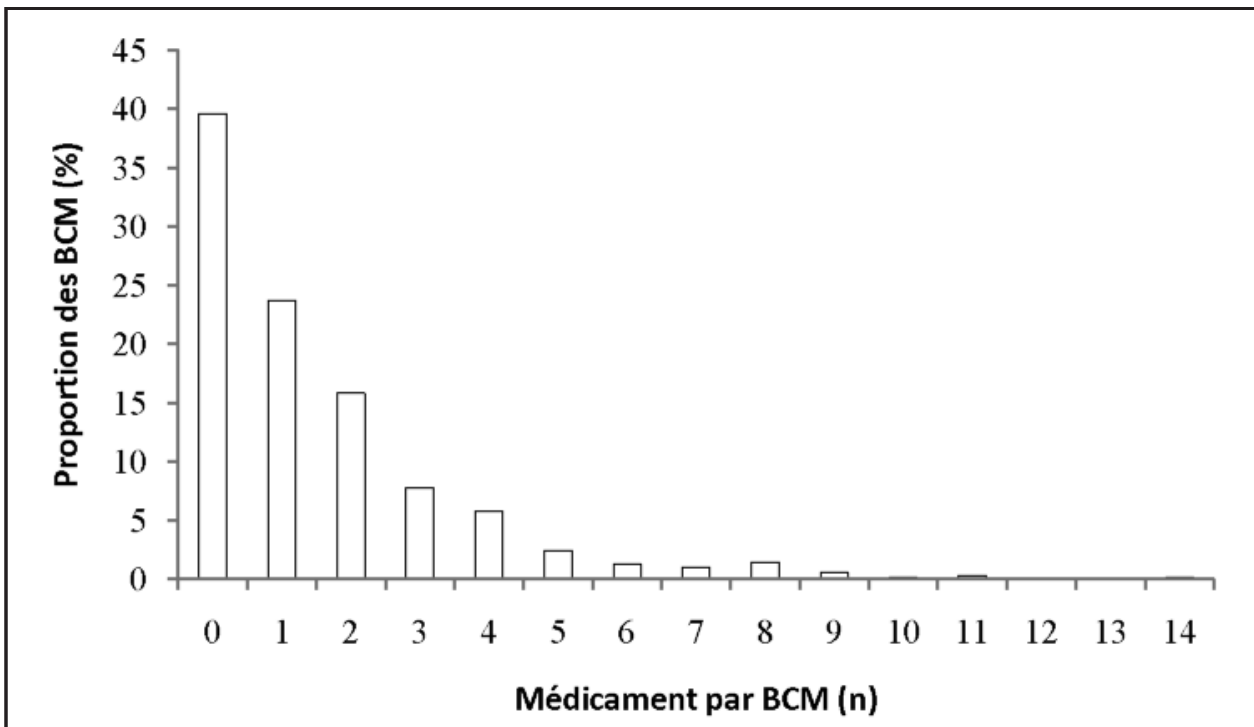

Figure 1. Proportion du nombre de médicaments contenus par bilan comparatif des médicaments (BCM).

pas accès au BCM à l'admission versé au dossier papier du patient, à moins que le $\mathrm{BCM}$ ne soit également une ordonnance.

\section{Réalisation du BCM}

La démarche du BCM a été introduite dans notre établissement en 2007, en préparation à une visite d'agrément intégrant notamment l'utilisation du BCM comme pratique organisationnelle requise. Des efforts ont été déployés afin de sensibiliser le personnel soignant à cette démarche. Nous avons mené une évaluation pré-post à l'échelle de l'établissement, confirmant la nécessité d'implanter le $\mathrm{BCM}^{22}$.

En avril 2008, 46\% des dossiers de patients admis comportaient un BCM à l'admission, contre $53 \%$ en novembre 2008, soit un mois avant la visite d'agrément en décembre 2008. De décembre 2010 à février 2011, le personnel soignant a transmis le BCM à l'admission dans une proportion de $28 \%$. Lors du suivi de l'étude pilote, nous avons développé un volet de l'intranet pharmacie permettant de publier en temps réel le taux de BCM remplis et télécopiés au département de pharmacie. Laffichage met en évidence les chambres-lits (dénominalisées) ne comportant pas de télécopie du BCM à l'admission, en vue de faciliter le travail de l'assistante, qui pouvait alors cibler les BCM à remplir et à télécopier. À partir de septembre 2011, tout le personnel soignant, et particulièrement les cadres et les assistantes-infirmières de chaque quart de travail, a été sensibilisé à la possibilité de consulter cette donnée. Dans la perspective d'une visite d'agrément annoncée pour décembre 2011, et dans le respect de la pratique organisationnelle requise, le taux moyen de patients détenant un BCM à l'admission est passé à plus de $80 \%$ à partir de novembre 2011, et ce taux s'est maintenu jusquà maintenant. La figure 2 illustre un exemple d'affichage web du taux de BCM remplis et télécopiés ainsi que le détail par unité de soins. La rétroaction en temps réel a été un facteur clé de succès dans ce projet et est implantée de façon permanente dans notre intranet.

De plus, l'implantation optimale du BCM à l'admission doit reposer sur une utilisation effective du formulaire par le personnel soignant et particulièrement les médecins et les pharmaciens aux unités d'hospitalisation et la transmission systématique du formulaire à l'équipe de la pharmacie. Si la sensibilisation réalisée jusqu’à maintenant et le fait qu'il s'agisse d'une Pratique organisationnelle requise en vertu des normes d'Agrément Canada ont contribué à notre succès, il aura fallu près de quatre années pour qu'un processus continu soit en place. Ainsi, la mise en place d'une nouvelle procédure de gestion en milieu hospitalier prend souvent plus de temps que prévu. Comme le souligne Balas et Boren, il faut en moyenne 17 années pour que les résultats de recherche clinique soient pleinement implantés en pratique clinique ${ }^{23}$.

Dans l'étude pilote, nous observons que la proportion de BCM par admission est plus élevée aux unités de soins comportant une présence quotidienne de pharmaciens décentralisés (p. ex. pédiatrie, multispécialité) qu’aux unités où les pharmaciens n'y vont que ponctuellement (p. ex. chirurgie). Toutefois, cette différence s'est estompée après l'étude pilote, compte tenu d'un taux de conformité supérieur à $80 \%$ pour l'ensemble de l'établissement.

La transmission des BCM à l'admission par télécopie facilite l'intégration au dossier pharmacologique informatisé et peut être utilisée par tous les pharmaciens lors de la validation ou même a posteriori sans que l'ensemble du dossier patient ne soit complètement informatisé. Il faut toutefois noter qu'il 


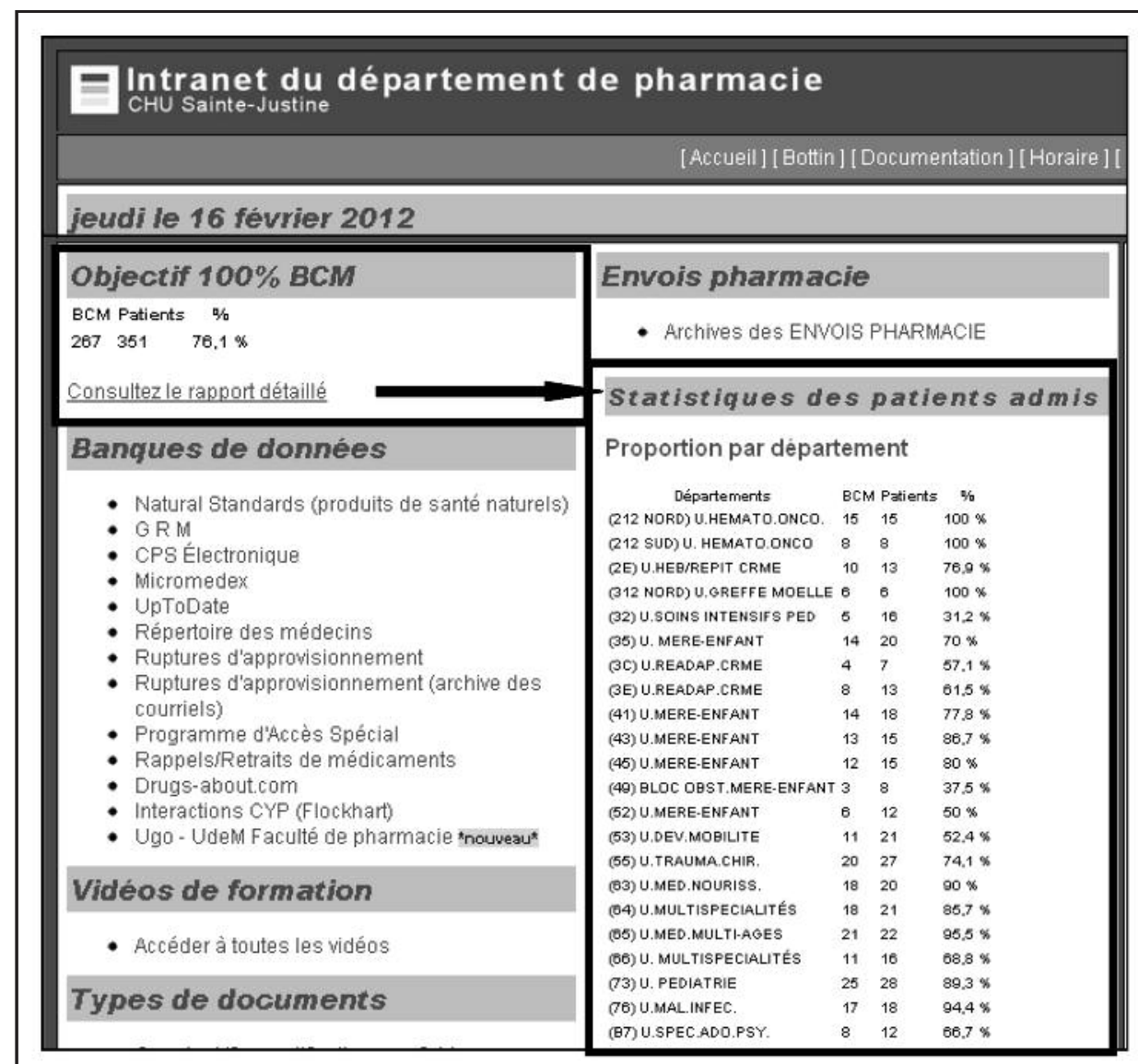

Figure 2. Affichage web du taux de bilan comparatif des médicaments (BCM) remplis et télécopiés ainsi que le détail par unité de soins.

s'agit d'une augmentation significative de l'information devant être traitée par le pharmacien centralisé (environ 40-50 nouveaux BCM à l'admission par jour dans notre hôpital).

Les améliorations apportées à la forme du BCM en vigueur (c.-à-d. ajout de colonnes et de choix de réponse) ont contribué à faire progresser la conformité de quatre des cinq critères évalués (c.-à-d. dose, voie, horaire d'administration et date/heure de dernière prise du médicament). Seule la variable «nom du médicament» présente un taux de conformité pratiquement inchangé. Ceci laisse entendre que l'élimination des sections inutiles et les modifications apportées facilitent le remplissage du formulaire par les usagers (médecins, infirmières, parents), surtout si ces derniers s'avèrent d'emblée moins à l'aise d'inscrire des renseignements sur les médicaments que les pharmaciens.

Bien que la majorité des enfants reçoivent moins de médicaments que les adultes, la démarche de BCM à l'admission est tout aussi importante pour ce groupe d'usagers ${ }^{2426}$. Pendant la durée de l'étude, les BCM à l'admission comportaient majoritairement un ou aucun médicament. Plusieurs hôpitaux québécois ont choisi de confier la tâche des
BCM à l'admission au personnel technique en pharmacie sous la supervision du pharmacien. Dans notre centre, compte tenu du caractère pédiatrique et du nombre plus limité de médicaments, il semble que l'infirmière et le médecin peuvent accomplir cette tâche avec la possibilité de consulter le pharmacien pour les cas plus complexes

Si notre équipe de pharmaciens reconnaît la compétence et la valeur ajoutée du pharmacien à la réalisation de BCM à l'admission, au transfert ou au congé, nous savons qu'actuellement, notre équipe n'est pas en mesure de réaliser tous ces BCM, compte tenu des ressources disponibles, de la pénurie persistante de ressources et des autres activités pharmaceutiques. Cette étude pilote met en évidence la capacité de mobiliser le personnel soignant, telles les infirmières, avec l'appui de pharmaciens décentralisés pour les cas complexes. Si cette approche s'avère réaliste en pédiatrie, où une majorité d'enfants sont rarement polymédicamentés, elle mérite d'être évaluée avec soin lorsqu'il s'agit de patients adultes, car la charge de travail inhérente au BCM peut être beaucoup plus importante. 


\section{Qualité des BCM à l'admission}

Bien que le processus de BCM soit formellement en place depuis 2007 dans notre établissement, il aura fallu apporter des améliorations au formulaire pour observer une augmentation substantielle de la conformité, notamment quant au statut allergique, à la personne ressource consultée, à la mention de produits de santé naturels, à la dose des médicaments, à la voie, à l'horaire d'administration et à la date/l'heure de la dernière prise. Si les outils informatiques ont pour mérite de mettre clairement en évidence les informations requises, les formulaires papier mal délimités induisent souvent une collecte et une retranscription incomplète des données.

$\mathrm{Au}$ Canada, les gouvernements provinciaux investissent massivement dans la mise en place de dossiers santé électroniques. Plusieurs autres pays ont adopté des orientations technologiques favorisant le déploiement d'un dossier santé électronique permettant aux cliniciens des hôpitaux de travailler plus efficacement. Nul doute qu'un tel outil contribuera significativement à la démarche de bilan comparatif des médicaments ${ }^{27,28}$. Toutefois, il incombe de rappeler qu'il ne suffit pas d'informatiser les données pour que les processus soient optimisés. La démarche du BCM vise non seulement à rendre disponible l'information mais aussi à détecter les divergences. En attendant le déploiement du dossier électronique, les professionnels de la santé doivent assurer la production d'une liste complète de tous les médicaments que le patient prend avant l'admission, en faisant appel au patient, en consultant les contenants et les listes qu'il a en sa possession, en contactant le pharmacien de ville ou d'autres soignants. De plus, les hôpitaux doivent établir un processus structuré qui permette de détecter toute anomalie entre ce qui est prescrit au patient et ce qu'il prend en réalité.

Cette étude comporte plusieurs limites. Il s'agit d'une étude pilote observationnelle s'étendant sur une courte période d'observation. Les BCM à l'admission dûment remplis et versés au dossier du patient mais non télécopiés n’ont pas été pris en compte. L'étude peut comporter un biais d'observation (c.-à-d. le personnel soignant était au courant de l'évaluation de sa pratique).

\section{CONCLUSION}

Le bilan comparatif des médicaments à l'admission est un processus structuré au cours duquel les professionnels de la santé travaillent en partenariat avec les patients, les familles et les soignants pour assurer la transmission d'une information exacte et complète sur les médicaments aux points de transition des soins du patient. La proportion de BCM transmis au département de pharmacie par admission était plus élevée dans les unités de soins comportant une présence quotidienne de pharmaciens décentralisés. De plus, l'inclusion d'espaces réservés et de cases à cocher sur un $\mathrm{BCM}$ s'est avérée une méthode efficace pour améliorer la conformité.

\section{Références}

1. Pratiques organisationnelles requises. Ottawa $(\mathrm{ON})$ : Agrément Canada; février 2011. Publié au: www.accreditation.ca/uploadedFiles/ ROP\%20Handbook\%20FR.pdf. Consulté le $1^{\text {er }}$ avril 2011. Sections concernant le bilan comparatif des médicaments.

2. Assuring medication accuracy at transitions in care. Patient Safety Solutions: Vol 1, Solution 6. Genève (La Suisse) : Organisation mondiale de la santé; 2007. Publié au: www.ccforpatientsafety.org/common/ pdfs/fpdf/presskit/PS-Solution6.pdf. Consulté le $1^{\text {er }}$ avril 2011).

3. Assuring medication accuracy at transitions in care: Standard Operating Protocol Fact Sheet. Genève (La Suisse) : Organisation mondiale de la santé. Publié au: https://www.high5s.org/pub/Manual/Training Materials/Assuring_Medication_Accuracy_at_Transitions_in_Care.doc. Consulté le $1^{\text {er }}$ avril 2011).

4. Manuel de certification des établissements de santé V2010. Saint-DenisLa-Plaine (France) : Haute Autorité de Santé, Direction de l'Amélioration de la Qualité et de la Sécurité des Soins; juin 2009. Publié au : www.hassante.fr/portail/upload/docs/application/pdf/2008-12/20081217_ manuel_v2010_nouvelle_maquette.pdf. Consulté le $1^{\text {er }}$ avril 2011.

5. Rozich JD, Resar RK. Medication safety: one organisation's approach to the challenge. J Clin Outcomes Manag 2001;8:27-34.

6. Cornish PL, Knowles SR, Marchesano R, Tam V, Shadowitz S, Juurlink $\mathrm{DN}$, et al. Unintended medication discrepancies at the time of hospital admission. Arch Intern Med 2005;165(4):424-429.

7. Bates DW, Cullen DJ, Laird N, Petersen LA, Small SD, Servi D, et al.; ADE Prevention study group. Incidence of adverse drug events and potential adverse drug events. Implications for prevention. JAMA 1995;274(1):29-34.

8. Leape LL, Brennan TA, Laird N, Lawthers AG, Localio AR, Barnes BA, et al. The nature of adverse events in hospitalized patients. Results of the Harvard Medical Practice Study II. N Engl J Med 1991;324(6):377-384.

9. Greenwald JL, Halasyamani LK, Greene J, LaCivita C, Stucky E, Benjamin $\mathrm{B}$, et al. Making inpatient medication reconciliation patient centered, clinically relevant, and implementable: a consensus statement on key principles and necessary first steps. Jt Comm J Qual Patient Saf 2010; 36(11):504-513, 481.

10. Paparella S. Medication reconciliation: doing what's right for safe patient care. J Emerg Nurs 2006;32(6):516-520.

11. Delate T, Chester EA, Stubbings TW, Barnes CA. Clinical outcomes of a home-based medication reconciliation program after discharge from a skilled nursing facility. Pharmacotherapy 2008;28(4):444-52.

12. Programs. CAPHC Patient Safety Collaborative [site web]. Ottawa (ON) Association canadienne des centres de santé pédiatriques; 2003-2008. Publié au: www.caphc.org/programs_patient_safety_pmrc.html. Consulté le 16 février 2012

13. Strunk LB, Matson AW, Steinke D. Impact of a pharmacist on medication reconciliation on patient admission to a veterans affairs medical center Hosp Pharm 2008;43:643-649.

14. Hayes BD, Donovan JL, Smith BS, Hartman CA. Pharmacist-conducted medication reconciliation in an emergency department. Am J Health Syst Pharm 2007;64(16):1720-1723.

15. Bruce Bayley K, Savitz LA, Maddalone T, Stoner SE, Hunt JS, Wells R. Evaluation of patient care interventions and recommendations by a transitional care pharmacist. Ther Clin Risk Manag 2007;3(4):695-703.

16. Beyea SC. Medication reconciliation: what every nurse needs to know. AORN J 2007;85(1):193-194

17. Gleason KM, Groszek JM, Sullivan C, Rooney D, Barnard C, Noskin GA. Reconciliation of discrepancies in medication histories and admission orders of newly hospitalized patients. Am J Health Syst Pharm 2004;61(16):1689-1695.

18. Chevalier BA, Parker DS, MacKinnon NJ, Sketris I. Nurses' perceptions of medication safety and medication reconciliation practices. Nurs Leadersh (Tor Ont) 2006;19(3):61-72.

19. Porter SC, Kohane IS, Goldmann DA. Parents as partners in obtaining the medication history. J Am Med Inform Assoc 2005;12(3):299-305.

20. Alemanni J, Atkinson S, Sauvé C, Bussières JF. Évaluation de lutilisation du bilan comparatif des médicaments à l'urgence. Pharmactuel 2010; 43(1):49-52.

21. Bussières JF. Services cliniques de pharmacie. Dans : Babich B, Bornstein $\mathrm{C}$, Bussières JF, Hall K, Harding J, Lefebvre P, et al., rédaction. Rapport 20092010 sur les pharmacies hospitalières canadiennes. Eli Lilly; 2010. p. 322. Publié au : www.lillyhospitalsurvey.ca/hpc2/content/2010_report/ chapter_bF\%20.pdf. Consulté le 1 ${ }^{\text {er }}$ avril 2011. 
22. Bédard P, Tardif L, Ferland A, Bussières JF, Lebel D, Bailey B, et al. A medication reconciliation form and its impact on the medical record in a paediatric hospital. J Eval Clin Pract 2011;17(2):222-227.

23. Balas EA, Boren SA. Managing clinical knowledge for health care improvement. Yearb Med Inform 2000:65-71.

24. Terry DR, Solanki GA, Sinclair AG, Marriott JF, Wilson KA. Clinical significance of medication reconciliation in children admitted to a UK pediatric hospital: observational study of neurosurgical patients. Paediatr Drugs 2010;12(5):331-337.

25. Stone BL, Boehme S, Mundorff MB, Maloney CG, Srivastava R. Hospital admission medication reconciliation in medically complex children: an observational study. Arch Dis Child 2010;95(4):250-255.

26. Gardner B, Graner K. Pharmacists' medication reconciliation-related clinical interventions in a children's hospital. It Comm I Qual Patient Saf 2009;35(5):278-282

27. Bassi J, Lau F, Bardal S. Use of information technology in medication reconciliation: a scoping review. Ann Pharmacother 2010;44(5):885-897.

28. Lau F, Kuziemsky C, Price M, Gardner J. A review on systematic reviews of health information system studies. I Am Med Inform Assoc 2010; 17(6):637-645.
Sophie Penfornis, D.Pharm, est Assistante de recherche, Unité de recherche en pratique pharmaceutique, CHU Sainte-Justine, Montréal (Québec) et Faculté de pharmacie, Université Lille 2, Lille, France.

Pascal Bédard, B. Pharm., M.Sc., est Pharmacien, Département de Pharmacie, CHU Sainte-Justine, Montréal, Québec.

Benoit Bailey, MD, FRCPC, est Directeur, Département de pédiatrie, service de I'urgence, CHU Sainte-Justine, Montréal, Québec.

Jean-François Bussières, B. Pharm., M.Sc., FCSHP, est Chef, Département de Pharmacie et Unité de recherche en pratique pharmaceutique, CHU Sainte-Justine, et professeur titulaire de clinique, Faculté de Pharmacie, Université de Montréal, Montréal, Québec.

\section{Adresse de correspondance :}

Jean-François Bussières

Département de pharmacie

Centre Hospitalier Universitaire Sainte-Justine

3175, chemin de la Côte Sainte-Catherine

Montréal QC H3T 1C5

Courriel : jf.bussieres@ssss.gouv.qc.ca

CSHP has introduced both Print and Print + Online pricing models for CJHP subscriptions. Print + Online CJHP is included as a benefit of CSHP membership. All prices are in Canadian funds.

La SCPH a établi une grille tarifaire pour l'abonnement à la copie imprimée du JCPH seulement et pour l'abonnement à la fois aux copies imprimée et électronique du journal. L'abonnement combiné est inclus dans les droits d'adhésion à la SCPH. Tous les prix sont en dollars canadiens.

\begin{tabular}{|l|l|l|}
\hline $\begin{array}{l}\text { Subscriber group / Groupe } \\
\text { d'abonnés }\end{array}$ & $\begin{array}{l}\text { Print only / Texte imprimé } \\
\text { seulement }\end{array}$ & $\begin{array}{l}\text { Print + Online copy } \\
\text { Texte imprimé } \\
\text { et exemplaire électronique }\end{array}$ \\
\hline $\begin{array}{l}\text { Nonmembers within Canada / } \\
\text { Non-membres au Canada }\end{array}$ & $\begin{array}{l}\$ 110.00 \text { per year, plus GST or HST } \\
110,00 \$ \text { par an, plus TPS ou TVH }\end{array}$ & $\begin{array}{l}\$ 150.00 \text { per year, plus GST or HST } \\
150,00 \$ \text { par an, plus TPS ou TVH }\end{array}$ \\
\hline USA / É.-U. & $\begin{array}{l}\$ 140.00 \text { per year } \\
140,00 \$ \text { par an }\end{array}$ & $\begin{array}{l}\$ 180.00 \text { per year } \\
180,00 \text { par an }\end{array}$ \\
\hline Foreign / Étranger & $\$ 180.00$ per year & $\$ 220.00$ per year \\
& 180,00 par an & $220,00 \$$ par an \\
\hline
\end{tabular}

More details can be found at www.cjhp-online.ca. If you would like to purchase a subscription, please fill out our CJHP 2012 Subscription Application Form, which can be found on the CJHP website.

Please direct any comments or questions to Colleen Drake, Publications Administrator,

at cdrake@cshp.ca.

Des détails supplémentaires sont fournis à www.cihp-online.ca. Si vous désirez vous abonner, veuillez remplir le formulaire d'abonnement au JCPH 2012. Vous pouvez l'obtenir en visitant le site Web du $\mathrm{JCPH}$. Pour tout commentaire ou toute question, veuillez vous adresser à Colleen Drake, agente des publications, en écrivant à cdrake@cshp.ca. 